PERFILES 9
E SATIHप3d

Perfiles Educativos

ISSN: 0185-2698

perfiles@unam.mx

Instituto de Investigaciones sobre la

Universidad y la Educación

México

Pantoja Castro, Julio César; Covarrubias Papahiu, Patricia

La enseñanza de la biología en el bachillerato a partir del aprendizaje basado en problemas (ABP)

Perfiles Educativos, vol. XXXV, núm. 139, 2013, pp. 93-109

Instituto de Investigaciones sobre la Universidad y la Educación

Distrito Federal, México

Disponible en: http://www.redalyc.org/articulo.oa?id=13225611005

Cómo citar el artículo

Número completo

- Más información del artículo

Página de la revista en redalyc.org

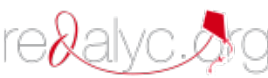

Sistema de Información Científica

Red de Revistas Científicas de América Latina, el Caribe, España y Portugal

Proyecto académico sin fines de lucro, desarrollado bajo la iniciativa de acceso abierto 


\title{
La enseñanza de la biología en el bachillerato a partir del aprendizaje basado en problemas (ABP)
}

\author{
Julio César Pantoja Castro* Patricia Covarrubias Papahiu***
}

Con base en los fundamentos constructivistas de la educación, el aprendizaje basado en problemas (ABP) se constituye como una opción viable para contrarrestar los problemas que conlleva la enseñanza tradicional de la ciencia. En tal sentido, se presenta una investigación cimentada en el ABP que muestra la promoción de habilidades de pensamiento necesarias para el aprendizaje significativo de contenidos de la biología en el bachillerato. Se trabajó con estudiantes de sexto semestre del Colegio de Ciencias y Humanidades, particularmente en una situación-problema que requirió de su análisis y solución a partir de los principios de la selección natural, y del apoyo de diversas estrategias didácticas. Se utilizó un diseño cuasi-experimental, con análisis estadísticos y cualitativos de los datos obtenidos. Los resultados sugieren que el ABP es una opción pedagógica para el aprendizaje significativo de contenidos de la Biología, o bien, como estrategia didáctica complementaria que potencia estrategias de enseñanza más tradicionales.

Based on the constructivist foundations of education, the problem-based learning offers an excellent option to thwart the problems entailed by the traditional teaching of sciences. This research presents an implementation of the problem-based learning strategies that shows the importance of promoting thinking abilities that are necessary for the significant learning of Biology contents in high school. The authors worked with high school seniors of the Colegio de Ciencias y Humanidades (UNAM, Mexico), and particularly in a situation-problem that required their analysis and solution based on the principles of natural selection, and the support of diverse didactic strategies. The authors made use of a quasi-experimental design with statistical and qualitative analysis of the gathered data. The results show that problem-based learning is a good pedagogical option to reach significant learning of the Biology contents or as a complementary teaching strategy that boosts more traditional teaching strategies.
Palabras clave

Aprendizaje basado en problemas

Estrategias de enseñanza

Bachillerato

Biología

Enseñanza de la biología

Selección natural

Keywords

Problem-based learning

Teaching strategies

High school

Biology

Teaching of Biology

Natural selection

Recepción: 18 de agosto de 2011| Aceptación: 19 de enero de 2012

* Maestro en Docencia para la Educación Media Superior en el campo de la Biología, Facultad de Estudios Superiores (FES)-Iztacala, UNAM.Profesordefinitivo Bdelaasignatura de Biología Ia IV en elColegiodeCienciasyHumanidades plantel Naucalpan. Líneas de investigación: procesos de enseñanza aprendizaje en el área de Biología. CE: jcpantojac (a) yahoo.com.mx

** Doctora en Pedagogía por la Facultad de Filosofía y Letras de la UNAM. Profesora de la carrera de Psicología e investigadora de la Facultad de Estudios Superiores (FES)-Iztacala, UNAM. Líneas de investigación: currículo, prácticas pedagógicas, procesos de enseñanza y aprendizaje. Publicaciones recientes: (2011), "Representaciones sobre la enseñanza. Una indagación en estudiantes universitarios”, Sinéctica, núm. 36, en: www.sinectica.iteso.mx; (2010), Construcción del conocimiento e identidad profesional, México, UNAM-FES Iztacala/Castellanos Editores. CE: papahiu@unam.mx 


\section{INTRODUCCIÓN}

El propósito del artículo es presentar una alternativa de enseñanza para el aprendizaje de los contenidos de la biología que se imparten en la educación media superior; esta alternativa por un lado soluciona varios de los problemas que la enseñanza tradicional presenta, y por otro, representa una muestra de cómo se pueden llevar a la práctica los principios pedagógicos constructivistas que fundamentan los actuales planes de estudio. Se expone para ello una investigación basada en los principios y procedimientos del método conocido como aprendizaje basado en problemas (ABP), en estudiantes del Colegio de Ciencias y Humanidades (CCH) de la UNAM.

\section{Algunos Problemas}

\section{EN LA ENSEÑANZA Y APRENDIZAJE DE LA CIENCIA}

Aquella idea ingenua según la cual sólo se necesita tener el conocimiento de tipo declarativo en un área específica de la ciencia para su enseñanza, es decir, conocer solamente los hechos, conceptos y principios que la caracterizan, o bien, aquella que decía que bastaba con aprender una serie de pasos preestablecidos que deben seguirse de forma lineal y rigurosa para hacer ciencia, han quedado atrás hace mucho tiempo, por lo menos en el discurso formal de lo educativo. Esto lo podemos constatar en las reformas curriculares e innovaciones promovidas desde la última década del siglo pasado, tanto en nuestro país como a nivel mundial, y en todos los niveles educativos.

En estas reformas curriculares e innovaciones pedagógicas, derivadas de las recomendaciones que han hecho algunos organismos nacionales e internacionales, en sus fundamentos pedagógicos se coloca a los estudiantes como los principales actores del proceso educativo, y se propone que "aprendan a aprender", en tanto la sociedad actual les plantea retos y desafíos en su capacidad para acrecentar sus conocimientos, y en la adquisición de nuevas formas de relacionarse e interactuar con ellos. De la institución educativa y del docente se espera diseñen experiencias de aprendizaje que permitan a los estudiantes desarrollar habilidades de pensamiento analítico, crítico, creativo o de resolución de problemas, que favorezcan su propia construcción del conocimiento y se orienten hacia la ciencia.

Sin embargo, en la mayoría de los escenarios educativos no se ha logrado trascender la enseñanza tradicional de las ciencias en la práctica. Desafortunadamente muchos de los cursos y programas se desarrollan con el fin de transmitir conocimientos que los estudiantes tienen que reproducir para obtener una calificación; habrá otros que alcanzan a promover hábitos o técnicas de estudio, proporcionándoles a los estudiantes un bagaje más o menos completo de destrezas, pero rara vez se les enseña a utilizarlas en los contextos idóneos. Como resultado, la generalidad de los estudiantes dedican la mayor parte del tiempo a memorizar nombres, datos, eventos, etcétera, que más tarde deben reproducir con fidelidad pero que terminan olvidando (Pozo, 1993).

Nuestra experiencia como docentes en educación media superior nos permite afirmar que estos problemas se presentan con mucha similitud en el caso particular del $\mathrm{CCH}$, en donde las prácticas de enseñanza en muchas ocasiones no logran materializar con oportunidad y propiedad los principios constructivistas que fundamentan su modelo pedagógico. Si bien existen experiencias aisladas de profesores que hacen uso de estos principios y de algunas estrategias didácticas diferentes a las tradicionales para promover la comprensión y aplicación de los contenidos disciplinarios de algunas asignaturas, lo cierto es que predomina la mera transmisión de conocimientos, la cual impide que los estudiantes alcancen los objetivos deseados que les permitirían el aprendizaje de la ciencia y la construcción del conocimiento en ella. 
Particularmente, los temas de las diferentes materias a desarrollar en el nivel medio superior son de naturaleza compleja, pues implican razonamientos e inferencias que hacen que los estudiantes vayan prescindiendo de sus percepciones individuales inmediatas y concretas para asir esquemas donde la abstracción aproxima el razonamiento a las formas más comunes del proceder de la ciencia. En este sentido, la exigencia es mayor para el desarrollo de actitudes más relacionadas con la precisión, la objetividad, la flexibilidad, la observación, la inferencia, la deducción, la traducción, la discusión y el manejo de argumentos; las habilidades de búsqueda e interpretación de datos y conceptos, la participación en equipos, el debate colectivo y el desarrollo de la capacidad crítica (Lomelí, 1991).

Con todo y estas exigencias que el bachillerato plantea a los estudiantes, se sigue dando mayor peso a los aprendizajes de tipo memorístico, desconectados de la vida cotidiana, lo que origina que el conocimiento, en muchas ocasiones, carezca de significado para el estudiante. Aunado a lo anterior, los estudiantes tienen una carga de ideas o preconcepciones que para el caso de la biología resultan de origen analógico ${ }^{1}$ a ésta, lo que da como resultado que los contenidos temáticos sobre procesos biológicos no sean aprehendidos significativamente por la mayor parte de ellos (Estévez, 2002).

Por tal motivo, el propósito del artículo es mostrar, mediante una investigación realizada con los fundamentos del aprendizaje basado en problemas (ABP), la forma en que el profesor puede organizar la enseñanza para que los estudiantes desarrollen las habilidades de pensamiento necesarias para aproximarse al conocimiento y a la ciencia de una manera constructiva, y que los capacite para continuar preparándose de manera autónoma, o bien, les permita acceder y adaptarse con mayor facilidad a los estudios superiores. Iniciaremos describiendo el contexto en el que se realizó la investigación.

\section{LA ENSEÑANZA Y EL APRENDIZAJE DE LOS CONTENIDOS DE BIOLOGÍA EN EL CCH}

Con respecto a los problemas que se han detectado en el desempeño escolar de los estudiantes, en las jornadas de balance académico que tuvieron lugar en octubre de 2004 en el CCH plantel Naucalpan, los profesores mencionaron que los estudiantes presentan dificultades para comprender los temas de las materias, especialmente los abordados en la materia de biología, cuyos contenidos son predominantemente abstractos. Además, muchos profesores no relacionan estos contenidos con aspectos cotidianos de la vida, por lo que carecen de significado y como consecuencia a los estudiantes les parecen aburridos y densos; esto hace que con frecuencia se dificulte su aprendizaje, los estudiantes se sientan frustrados e incluso, en ocasiones, que abandonen la materia (CCH, 2004: 4).

Un ejemplo de lo anterior es lo reportado por De Manuel y Grau (1996), quienes advierten que un número importante de estudiantes tiene una falsa idea con respecto al mecanismo evolutivo en tanto lo explican ya sea por la necesidad de un cambio por parte del organismo, o por el uso o falta de uso de un órgano, e inclusive lo llegan a entender como la búsqueda de una perfección adaptativa por parte de los seres vivos.

Al parecer, el origen de esta concepción se encuentra en una mezcla de sentido común y empirismo. Llama la atención que la mayoría de los estudiantes emplean el concepto de adaptación, por ejemplo, desde el significado que se le da en el contexto cotidiano, es decir, como el esfuerzo que realiza un individuo para cambiar; y no en el sentido científico

1 Analógico en el sentido de que la transferencia será negativa cuando las preconcepciones sean erróneas con respecto al nuevo conocimiento (dominio fuente contra dominio objetivo). Esto conlleva a que las estructuras críticas de ambos dominios no sean equivalentes, y por tanto incorrecto el realizar extrapolaciones. Véase Diccionario enciclopédico (1995), México, Grijalbo. 
que se le asigna actualmente al mecanismo evolutivo, donde la adaptación se correlaciona con los genes contenidos en una población - poza génica - y, por otra parte, con factores ambientales y/o presiones selectivas ejercidas por otros organismos. Aquellos alelos -las variantes que presenta un gen- que proveen de alguna ventaja de sobrevivencia a los individuos que los poseen, serán seleccionados, y a través del tiempo estos alelos aumentan en la población, mientras aquellos que no den una ventaja adaptativa disminuyen su frecuencia. Es lo que se conoce como selección natural (De Manuel y Grau, 1996; Morrone, 2005). Preocupa, por lo tanto, que los estudiantes conserven concepciones erróneas en el ámbito escolar.

La materia de biología debe lograr que en la cultura básica del bachiller se incorporen conocimientos, habilidades intelectuales, actitudes y valores que favorezcan una interpretación lógica, racional y mejor fundamentada de la naturaleza, que disminuya la incidencia del pensamiento mágico y doctrinario como explicación del mundo natural, y que la interacción del alumno con la sociedad, la tecnología y el ambiente sea más consciente y responsable. Debe dotar al estudiante de los conocimientos y habilidades intelectuales que le permitan acceder por sí mismo a las fuentes del conocimiento, y más en general, de la cultura $(\mathrm{CCH}$, 2006). En otras palabras, crear las condiciones, a través del planteamiento de situaciones problema que impliquen la necesidad de manipular el significado de los conceptos, de las controversias propiciadas a partir del trabajo en grupo, y de la discusión general en el aula, para que el alumnado pueda expresar qué hay detrás de las simples etiquetas verbales de las palabras (De Manuel y Grau, 1996).

La concepción constructivista del aprendizaje escolar, que fundamenta al modelo pedagógico del $\mathrm{CCH}$, sustenta la idea de que la finalidad de la educación es promover los procesos de crecimiento personal del alumno en el marco de la cultura de grupo, aplicando actividades intencionales, planificadas y sistematizadas por el profesor, que logren propiciar en el estudiante una actividad mental constructivista; esto es, la realización de aprendizajes significativos que enriquezcan el conocimiento del mundo físico y social, potenciando así su crecimiento personal. De esta manera, los tres aspectos clave que debe favorecer el proceso instruccional son: el logro del aprendizaje significativo, la memorización comprensiva de los conocimientos escolares y la funcionalidad de lo aprendido (Coll, 1997).

En tal sentido, el ABP constituye una alternativa para la enseñanza de la biología, pues se distancia de la enseñanza de tipo enciclopedista - generalmente centrada en la disciplina, con escasa pertinencia social y personal-, y coloca en el centro a la persona que aprende, lo que permite a los estudiantes desarrollar su pensamiento formal.

\section{EL ABP COMO ALTERNATIVA PARA LA ENSEÑANZA DE LA SELECCIÓN NATURAL}

Las diversas modalidades que adopta el ABP son aportaciones de Piaget, Ausubel y Vigotsky y sus teorías constructivistas del aprendizaje. Se cita como antecedente importante del ABP el llamado "método de entrenamiento en investigación”, que desarrolló en los años sesenta Richard Schuman, con fundamento en el aprendizaje por descubrimiento de Jerome Bruner, y en los principios del razonamiento inductivo aplicado al método científico. En la educación es reconocido el papel pionero de la escuela de medicina de la Universidad de MacMaster de Canadá, así como el liderazgo e influencia de instituciones como la Harvard Business School y la Harvard Medical School en la aplicación del ABP; desde entonces el $\mathrm{ABP}$ es una herramienta que ha resultado útil en muchas partes del mundo, incluyendo América Latina y México, y en especial la Universidad Nacional Autónoma 
de México, la Universidad de Colima y el Instituto Tecnológico y de Estudios Superiores de Monterrey (Olivares, 2001; Díaz Barriga y Hernández, 2002; Díaz Barriga, 2006).

El ABP es concebido como un desafío para el estudiante; contribuye a que éste "aprenda a aprender" por medio de un problema real que plantea un conflicto cognitivo, es decir, una pérdida del equilibrio en sus esquemas de pensamiento; le ayuda a buscar respuestas, a plantearse interrogantes, a investigar, a descubrir, es decir, a aprender. En el ABP un equipo de estudiantes se reúne para buscar una solución (se dice que es el método de aprendizaje del adulto), por lo que se promueve el desarrollo de una cultura de trabajo colaborativo en donde se involucra a todos los miembros del grupo en el proceso de aprendizaje. El ABP permite que el grupo adquiera los conocimientos necesarios para enfrentarse al problema retador (Sánchez, 2002; Morales y Landa, 2004). En otras palabras, promueve la interrelación de los conocimientos previos de los estudiantes con los nuevos, mediante relaciones significativas que los ayudan a la maduración del pensamiento formal; que los llevan no sólo a aprender cómo utilizar determinados procedimientos, sino cuándo y por qué pueden ser utilizados (reflexión), lo que favorece los procesos cognitivos para la resolución de tareas y facilita el aprendizaje significativo (Monereo, 1999).

En síntesis, algunos beneficios del ABP que lo hacen atractivo para aplicarse en la enseñanza de contenidos científicos son: promueve una cultura de trabajo cooperativo en el que participan todos los miembros de un grupo; promueve también el desarrollo de habilidades interpersonales, lo que permite a los estudiantes ir adquiriendo los conocimientos para un desarrollo intelectual, científico, cultural y social; favorece los procesos de pensar y aprender, de forma consciente; promueve el pensamiento crítico. Requiere, por lo tanto, de apoyarse en técnicas didácticas que favorezcan el desarrollo de estas habilidades, así como las de autoevaluación, a partir de procesos metacognitivos.

Al considerar los problemas planteados y los objetivos propuestos por el $\mathrm{CCH}$ para la asignatura de biología, elegimos el aprendizaje basado en problemas (ABP) como estrategia pedagógica para el aprendizaje de sus contenidos, especialmente los relacionados con la selección natural, en donde los estudiantes presentan dificultades con la interpretación de los procesos adaptativos y de la herencia; como ya mencionamos, muchos llegan al aula con preconcepciones cuyo origen frecuentemente se debe a que fueron construidas a partir del sentido común y del empirismo. Estas concepciones han de ser enfrentadas y utilizadas como punto de partida para discusiones grupales, centradas en problemas conocidos por la mayoría de los estudiantes; en este proceso el profesorado constituye uno de los elementos de la confrontación, en la medida en que aporta informaciones que favorecen el cambio, es decir, debe de crear una ruptura que permita la reorganización de los campos del conocimiento. Esto se consigue planeando estrategias didácticas que tengan como componente esencial la discusión de problemas que conecten la ciencia con la sociedad, es decir, que se deriven de contextos cotidianos que propicien situaciones de aplicación en casos relacionados con experiencias vividas por los estudiantes (De Manuel y Grau, 1996).

El propósito de la investigación que a continuación detallamos fue aplicar el ABP con la intención de favorecer la construcción del conocimiento significativo de contenidos relacionados con la biología.

\section{DESCRIPCIÓN DE LA INVESTIGACIÓN}

\section{Objetivo}

Evaluar la influencia del ABP en el aprendizaje de contenidos sobre selección natural en estudiantes de sexto semestre del CCH Naucalpan a partir de su desempeño académico, de su 
participación en equipos de trabajo y de su motivación por la estrategia didáctica empleada. ${ }^{2}$

\section{Diseño}

Se utilizó un diseño de tipo cuasi-experimental con un esquema de pre-prueba-intervención-post-prueba con grupos intactos: uno experimental (con 21 estudiantes entre los 16 y 17 años) y otro control (con 18 estudiantes entre los 16 y 17 años). A pesar de que no se pudieron controlar deliberadamente variables como la inteligencia, las aptitudes, las actitudes, los intereses, el promedio, los hábitos y métodos de estudio, la clase social, el ambiente cultural, etc. (Hernández et al., 2003), se aseguró la equivalencia inicial de los grupos en características básicas: ambos eran del sexto semestre del turno matutino, con el mismo promedio de edad y con conocimientos previos similares sobre el tema trabajado (Bisquerra, 1996; Hernández et al., 2003).

También se considera un estudio exploratorio (Stake, 1999), debido a que, si bien se ha aplicado el ABP para la enseñanza de temas relacionados con la biología tanto en el $\mathrm{CCH}$ como en otras instituciones y en otros países, ésta sería una primera experiencia para el tema de selección natural y procesos de evolucion, y una nueva oportunidad para contribuir al conocimiento de la estrategia del ABP

Al grupo experimental se aplicó el ABP durante nueve sesiones de dos horas cada una, en las que se abordó el tema "fuerzas evolutivas y sus consecuencias", de la materia de Biología IV que se imparte en el sexto semestre de bachillerato, relacionando la selección natural como un factor en los procesos de evolución.

Como estrategia de ABP se planteó a los estudiantes una situación-problema que se presenta frecuentemente en la vida real, en un formato de narrativa o historia que contenía una serie de atributos que mostraban complejidad y multidimensionalidad: la situación de una persona que habita en el valle de México, con un padecimiento faringoamigdalítico y aumento de la resistencia bacteriana a la penicilina como consecuencia de la automedicación. Se solicitó a los estudiantes que desarrollaran propuestas conducentes a su análisis y solución con la intención de que experimentaran la complejidad, ambigüedad, incertidumbre y falta de certeza que enfrentó el actor del caso. El propósito de este tipo de escenarios en el ABP consiste en que los estudiantes se apropien o se involucren en el caso, expresen sus emociones y activen sus valores; que discutan con argumentos, generen y sustenten ideas propias sin dejar de lado el punto de vista de los demás, con actitud de apertura y tolerancia ante las ideas de los otros. De esta manera pueden identificar en el problema sus componentes clave y así construir una o más opciones para afrontar y solucionar dicha situación (Díaz Barriga, 2006).

Al grupo seleccionado como control también se le aplicaron la pre-evaluación y postevaluación, pero el tema de selección natural lo impartió el titular de la materia, quien trabajó sus contenidos con técnicas didácticas muy acordes con el modelo educativo del $\mathrm{CCH}$.

El modelo educativo del $\mathrm{CCH}$, al considerar al estudiante como sujeto de su aprendizaje, de su formación y de su cultura, adoptó desde sus orígenes los principios de una educación moderna, considerando al estudiante como individuo capaz de captar por sí mismo el conocimiento y sus aplicaciones. En cada generación del CCH se "busca que los egresados bachilleres sepan pensar por sí mismos, se expresen adecuadamente, realicen cálculos y posean los principios de una cultura científica y humanística que sirva para relacionarlos con las diversas situaciones que se les presentan en su vida" (CCH, 1996: 46). En este sentido, el trabajo del docente del Colegio debe consistir en dotar a sus alumnos de los instrumentos

2 Los resultados de esta investigación se presentaron en la ponencia "Aprendizaje Basado en Problemas (ABP): Una alternativa para la enseñanza de la Biología en Educación Media Superior”, en el Congreso Internacional de Educación: Currículum, en la Universidad Autónoma de Tlaxcala, Tlaxcala, 2009, y en el Primer Congreso Latinoamericano de Ciencias de la Educación en la Universidad Autónoma de Baja California, Mexicali, 2010. 
metodológicos necesarios para que posean los principios de una cultura científica y humanística, promoviendo un conocimiento auténtico y una formación de actitudes tal que propicie que los estudiantes participen activamente en el proceso educativo bajo la guía del profesor, quien intercambia experiencias con sus colegas en diferentes espacios académicos. En el modelo educativo del CCH se concibe al profesor no sólo como el transmisor del conocimiento, sino como un compañero responsable y propositivo de experiencias de aprendizaje, capaz de apoyar el proceso de adquisición de nuevos conocimientos en los estudiantes, y de toma de conciencia acerca de cómo proceder mediante el acopio de información y de procesos de reflexión rigurosos y sistemáticos (CCH, 1996).

En este sentido, queda claro que las técnicas didácticas sugeridas por el modelo educativo del CCH se distancian del modelo tradicional de la enseñanza en tanto su intención es promover un aprendizaje dinámico en los estudiantes mediante su participación activa, tanto en el aula como en los trabajos de investigación y de laboratorio.

Del mismo modo, para lograr que los estudiantes sean capaces de aprender a aprender, aprender a hacer y aprender a ser, con sentido crítico, conscientes de sus razones, de sus saberes, de sus actuaciones y de sus valores, el modelo prevé que el profesor deberá desempeñarse como orientador en el aprendizaje, y será capaz de favorecer la autonomía del aprendizaje y la capacidad de juicio crítico del estudiante; asimismo, contempla que el docente esté dispuesto a aprender mientras enseña, y a seguir aprendiendo. Para ello se deben proponer actividades de aprendizaje en las que los estudiantes sean los protagonistas de la construcción de sus conocimientos, en un proceso colectivo donde el trabajo personal se vea enriquecido y apoyado por sus compañeros y el profesor (CCH, 1996).

En nuestro caso, como se dijo antes, el profesor titular del grupo control abordó el tema de selección natural de manera muy cercana a los planteamientos constructivistas del modelo educativo del CCH: organizó y desarrolló actividades y problemas que despertaran el interés y estuvieran acordes con la etapa de desarrollo de los estudiantes, utilizando técnicas didácticas como lluvia de ideas, resúmenes, mapas y redes conceptuales, analogías, ilustraciones y prácticas de laboratorio.

Varias de estas técnicas también fueron empleadas en el grupo experimental en tanto el ABP las contempla como apoyo para la solución de la situación-problema planteada a los estudiantes; de esta manera se reforzó el aprendizaje significativo del tema de selección natural, así como el desarrollo de habilidades metacognitivas y de interacción social para generar una cultura de trabajo cooperativo entre todos los estudiantes del grupo.

Dado lo anterior, la diferencia entre el grupo experimental y el grupo control radicó esencialmente en la situación-problema planteada a los estudiantes del grupo experimental como condición básica del ABP.

\section{Instrumentos de recolección de datos}

Se diseñaron varios instrumentos con base en lo que se quería evaluar a lo largo de las sesiones; no sólo se utilizaron instrumentos que evaluaran los conocimientos sobre la disciplina, principalmente a partir del desempeño académico de los estudiantes (tanto individual como en equipo), sino también las actitudes y valores necesarios para un adecuado desenvolvimiento en el salón de clases, e indispensables para que se logren aprendizajes significativos. Esta variedad de instrumentos permitió integrar varias dimensiones para su análisis, como el aprendizaje, la motivación y el trabajo en equipo, con las cuales se intentó llegar a una perspectiva unificadora entre los datos cuantitativos y los datos cualitativos obtenidos (Bisquerra, 1996; Stake, 1999; Hernández et al., 2003). Esta diversidad de datos fue de gran ayuda para profundizar sobre la influencia del ABP en el desempeño de los estudiantes. 
Algunos de los instrumentos fueron disenados ex profeso y otros se adaptaron para los propósitos de la investigación:

a) Cuestionario de conocimientos sobre selección natural: se elaboró específicamente para evaluar los conocimientos previos (pre-prueba) y los conocimientos adquiridos (post-prueba) tanto por el grupo experimental como por el grupo control en el tema de selección natural. Los demás instrumentos sólo se emplearon en el grupo experimental.

b) Inventarios sobre el desempeño académico individual de los estudiantes: se adaptaron dos inventarios elaborados por el Instituto Tecnológico y de Estudios Superiores de Monterrey (ITESM, 2004) para efectos de esta investigación, uno utilizado por el profesor y otro resuelto por cada uno de los estudiantes de cada equipo. Aunque con formatos diferentes, ambos inventarios se utilizaron para evaluar la interacción cooperativa de los estudiantes, sus competencias comunicativas, y su autonomía (trabajo y responsabilidad).

El primer inventario fue evaluado por el profesor a lo largo de cada una de las sesiones, mientras que el segundo se aplicó en la última sesión por los propios estudiantes. En él se consideraron de forma global las nueve sesiones en las que se trabajó con el ABP; cada integrante evaluó a sus compañeros de equipo. La utilización de los dos inventarios permitió obtener diferencias y similitudes entre la evaluación realizada por el profesor y la evaluación realizada por los estudiantes.

c) Diferencial semántico: fue desarrollado por Osgood et al. (1957, cit. por Hernández et al., 2003). Se trata de una escala de intervalo cuantitativa que consiste en una serie de adjetivos extremos. Una versión de ésta fue elaborada por el ITESM (2004), la cual se retomó para esta investigación con algunas modificaciones para que los propios estudiantes estimaran si las siguientes habilidades habían sido promovidas por el ABP: el aprendizaje independiente, el razonamiento crítico, la comunicación interpersonal, el procesamiento de la información, la formación personal y la autoevaluación. Se aplicó al final de las nueve sesiones, y sus resultados permitieron valorar la actitud favorable o desfavorable de los estudiantes hacia las habilidades que promueve el ABP.

d) Cuestionario de opiniones de los estudiantes sobre el $A B P$ : se diseñó específicamente para explorar con preguntas abiertas y con mayor profundidad la opinión de los estudiantes con respecto a algunos atributos del contexto durante la aplicación de la estrategia del ABP y la forma en que éstos incidieron en su participación. Los atributos evaluados fueron: ambiente generado en el aula, la importancia del ABP como estrategia para su participación, la actuación por equipo a lo largo de las sesiones, la motivación que les pudo haber generado y el valor de la estrategia ABP como herramienta de trabajo grupal para activar valores y habilidades en los estudiantes. Las respuestas fueron diversas y complementarias a la información obtenida en los otros instrumentos. Los resultados de este instrumento requirieron de un análisis cualitativo y la construcción de categorías analíticas que incorporen las opiniones compartidas por los estudiantes (Hernández et al., 2003). 


\section{Resultados}

Los resultados obtenidos en cada uno de los instrumentos aplicados en la fase de la preprueba y post-prueba del grupo control y grupo experimental, así como los aplicados en la fase de intervención con el grupo experimental, fueron los siguientes:

\section{Aplicación de estadístico al cuestionario de conocimientos sobre selección natural} El cuestionario de conocimientos se estructuró de acuerdo al programa indicativo de Biología IV del CCH (Programa de Estudios de Biología I a IV, 2004), del Área de Ciencias Experimentales. Incluyó un conjunto de preguntas o reactivos que se consideraron relevantes para el conocimiento y entendimiento de los temas. La estructura del cuestionario se dividió en tres partes: la primera parte se centró en indagar si los estudiantes comprendían ciertos conceptos o reconocían información sobre aspectos de selección natural y procesos de evolución: población, genes, adaptación, mutación, ambiente y variabilidad se calificaron de forma cuantitativa; en la segunda parte se evaluaron habilidades de tipo cognoscitivo procedimental a partir de una lista de palabras libres que los estudiantes tenían que interpretar y asociar de manera coherente; en la tercera parte los estudiantes tenían que contestar preguntas abiertas encaminadas a la aplicación de los conocimientos adquiridos por medio de una explicación de un suceso acontecido en su vida diaria y lo visto en la estrategia aplicada para el tema de selección natural y procesos de evolución. La segunda y tercera partes se evaluaron de manera cualitativa para después asignar una calificación.

Con el fin de conocer si las diferencias encontradas entre el grupo experimental y el grupo control, en el cuestionario de conocimientos, fueron significativas, se aplicaron pruebas estadísticas. a) Prueba de normalidad de KolmogorovSmirnov para dos muestras. Esta prueba se utilizó para comprobar que en las dos muestras (grupo control y experimental) los datos de la pre-prueba y post-prueba se encontraban distribuidos normalmente; es decir, si los datos se encontraban bajo la curva, para poder aplicar una prueba " $t$ " para datos correlacionados o apareados, con el fin de averiguar, a partir de los datos homogéneos de la pre-prueba y postprueba del grupo experimental y control, si la estrategia del ABP influyó más en los aprendizajes de los estudiantes del grupo experimental que en los estudiantes del grupo control.

Los resultados obtenidos de la prueba de normalidad de Kolmogorov-Smirnov aplicada al grupo experimental y control fueron: para el grupo experimental se obtuvo una ${ }^{*} \mathrm{P}_{\text {obt }}$ de 0.85 en la pre-prueba y una ${ }^{*} \mathrm{P}_{\text {obt }}$ de 0.94 para la post-prueba; estos valores estuvieron por arriba del valor del nivel de significancia $(=0.05)$ que fue de $P \alpha 0.31$, mientras que para el grupo control se obtuvo una ${ }^{*} \mathrm{P}_{\text {obt }}$ de 0.84 en la pre-prueba y una $* \mathrm{P}_{\text {obt }}$ de 0.51 en la postprueba, valores que también estuvieron por arriba del nivel de significancia $(\alpha=0.05)$ cuyo valor fue de *P 0.29, de esta manera: si $\mathrm{P}_{\mathrm{obt}} \geq \mathrm{Pa}$ se acepta $\mathrm{Ho}$.

De lo anterior se concluye que los datos de la pre-prueba y post-prueba tanto del grupo experimental como del grupo control se encontraron distribuidos normalmente, con una media de 0 , y una varianza aproximadamente de 1 (Daniel, 1993; Dytham, 2001; Exteberria et al., 1991): *P=probabilidad; $\mathrm{obt}=$ obtenida; $\alpha=$ nivel de significancia-tablas.

b) Prueba de t para muestras independientes. Al tener las dos muestras poblacionales - grupo experimental y grupo control- una distribución normal, se procedió a la aplicación de una prueba de ${ }^{\star} t$ para muestras independientes, con el propósito de conocer si existían diferencias en cuanto al conocimiento sobre selección natural y procesos de evolución antes 
de la enseñanza entre el grupo experimental y el grupo control; esta prueba permitió comparar las dos medias con las variables numéricas de las dos poblaciones.

Los resultados nos indican que la ${ }^{*} t_{\text {obt }}$ para comparar la pre-prueba entre ambos grupos fue de -0.130 , menor al nivel de significancia $(\alpha=0.05)$ que fue de ${ }^{\star} t \alpha-2.05$, por lo que:

$$
\mathrm{t}_{\text {obt }}=-0.130 /</ \mathrm{t} \alpha=-2.05 /
$$

Conservamos $\mathrm{Ho}$, y se concluye que no hubo diferencias significativas entre el grupo experimental y el grupo control en cuanto a los conocimientos antes de la enseñanza (Daniel, 1993; Dytham, 2001).

${ }^{*} \mathrm{t}=\mathrm{t}$ de Student; $\mathrm{t}_{\mathrm{obt}}=\mathrm{t}$ obtenida; $\mathrm{t} \mathrm{a}=\mathrm{t}$ de tablas (nivel de significancia).

c) Prueba de t para muestras apareadas. Esta prueba se utilizó para conocer la efectividad de un tratamiento - antes y después-, que en este caso fue la influencia de la estrategia $\mathrm{ABP}$ en el desempeño académico, comparada con una clase impartida de acuerdo al programa de BiologíaIV delCCH(Daniel, 1993; Dytham,2001).

Para el grupo experimental la $t_{\text {calculada }}$ fue de $-10.71 / \geq /$ a la ta -1.74 : se rechaza Ho.

Para el grupo control la $t_{\text {calculada }}$ fue de -7.99 / $\geq$ / a la ta -1.73 : se rechaza Ho.

En ambos casos se rechaza la hipótesis nula (Ho), se acepta la hipótesis alterna (Ha), y se concluye que los resultados de la prueba de $t$ para muestras apareadas, tanto en el grupo experimental como en el grupo control, presentaron, diferencias significativas entre las calificaciones de la pre-prueba y la postprueba, es decir, ambos grupos mejoraron su desempeño académico después de la enseñanza impartida con ABP (grupo experimental) y después de la enseñanza impartida de acuerdo al programa (grupo control).

Aunque no se obtuvieron diferencias estadísticamente significativas entre el grupo experimental y el de control en cuanto al desempeño global, sí se encontraron cambios a favor del grupo experimental en lo que corresponde a calificaciones aprobatorias. Esto se confirmó al aplicar la regresión lineal (Gráfica 1), en donde las dispersiones de los datos indican que fueron más los estudiantes del grupo experimental que obtuvieron calificaciones aprobatorias, y cuyas calificaciones fueron más altas en la postprueba, en comparación con las que obtuvo el grupo control. En la Gráfica 1 se aprecia que tanto el grupo experimental (0.91) como el grupo control (0.99) partieron en la pre-prueba (eje y) de conocimientos similares al aplicar los mínimos cuadrados, mientras en la post-prueba (eje $\mathrm{x}$ ), el grupo experimental obtuvo mejores resultados que el grupo control (1.8 contra 1.12), aunque esto es sólo una tendencia.

\section{Gráfica 1. Diagrama de dispersión de} la pre-prueba y post-prueba tanto del grupo experimental como del grupo control, en torno a las rectas de regresión

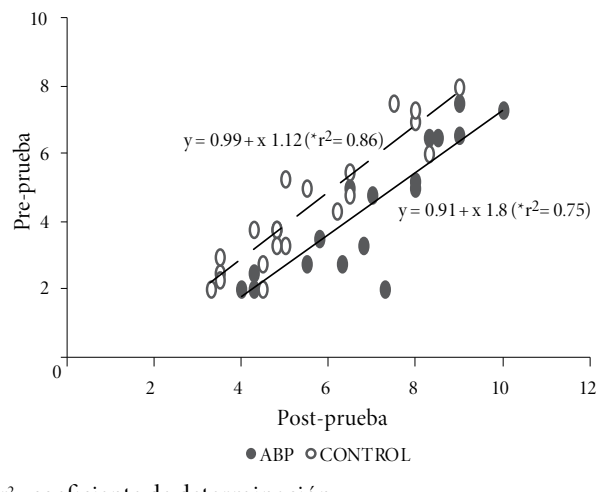

$* \mathrm{r}^{2}=$ coeficiente de determinación

\section{Evaluación del cuestionario de}

\section{conocimientos sobre selección natural}

Obtenidos los resultados estadísticos, se procedió a la evaluación del cuestionario de conocimientos aplicado a los dos grupos. Se obtuvo la suma total de las respuestas en las tres preguntas de todos los cuestionarios aplicados y se calcularon los porcentajes para su análisis.

En el Gráfica 2 se observa un cambio importante en el grupo experimental, pues de un 11.1 por ciento en la pre-prueba se pasó a un 48 por ciento en la post-prueba, es decir, 37 por ciento más estudiantes pasaron a formar parte 
de la categoría "buena" al contestar correctamente las tres preguntas. De la misma manera, pero ahora con una reducción importante, tenemos a la categoría "muy baja", debido a que 37 por ciento de estudiantes no contestaron correctamente la pre-prueba; este porcentaje, sin embargo, disminuyó hasta un 1.8 por ciento en la post-prueba, es decir, hubo una diferencia de 35.2 por ciento. En las categorías "aceptable" y "baja" los cambios fueron mínimos, pero positivos, en comparación con las categorías anteriores, puesto que para la categoría "aceptable" se dio un incremento de 9.3 por ciento (preprueba 20.4 por ciento y post-prueba 29.7 por ciento) y para la categoría "baja" un decremento de 11.1 por ciento (pre-prueba 31.5 por ciento y post-prueba 20.4 por ciento).

Si comparamos los resultados del grupo experimental (Gráfica 2) con los del grupo control (Gráfica 3), tenemos que en este último los cambios no fueron tan importantes, a excepción de la categoría "muy baja", en donde la diferencia fue de 23.4 por ciento: de tener 31.7 por ciento en la pre-prueba, se pasó a 8.3 por ciento de estudiantes que no contestaron adecuadamente las tres preguntas en la post-prueba. La mayoría pasaron a la siguiente categoría ("baja"), y algunos a la categoría "aceptable".

Gráfica 2. Porcentajes globales de las respuestas en las tres preguntas de la pre-prueba y post-prueba del grupo experimental (ABP)

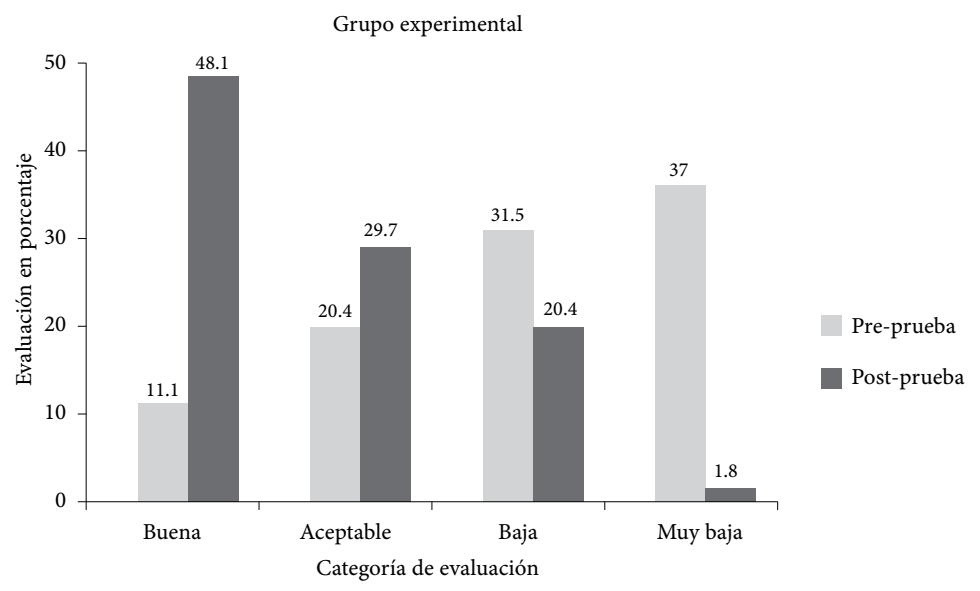

Gráfica 3. Porcentajes globales de las respuestas en las tres preguntas de la pre-prueba y post-prueba del grupo control

Grupo control

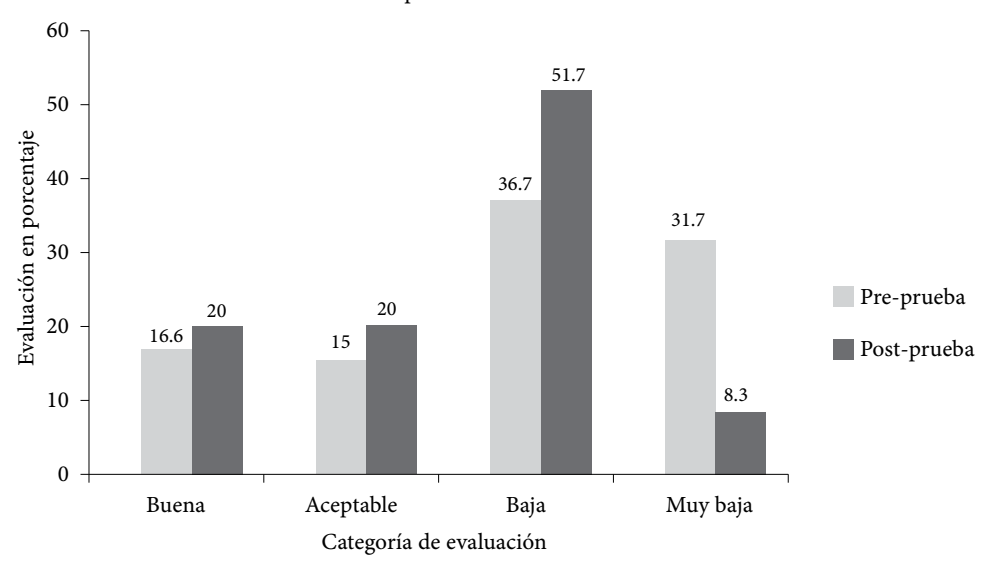


En síntesis, el grupo experimental calificó mejor las tres preguntas del cuestionario de conocimiento que el grupo control.

\section{Evaluación del desempeño académico individual de los estudiantes}

Los porcentajes obtenidos en las tres dimensiones del inventario del desempeño académico individual evaluadas por el profesor durante las nueve sesiones de la aplicación del ABP (Gráfica 4), y el inventario en el que los estudiantes evaluaron a cada uno de sus compañeros del equipo (Gráfica 5), muestran que para la dimensión de competencia comunicativa, los estudiantes evaluaron mejor a sus compañeros que el profesor en la categoría "distinguida" (64 por ciento los estudiantes y 51.8 por ciento el profesor), sin ser muy amplia la diferencia.

En la dimensión de interacción cooperativa, el profesor evaluó la actuación de los estudiantes en la categoría "satisfactoria" en 56.7 por ciento de los casos; en contraste, los estudiantes evaluaron esta misma categoría sólo en un 37.5 de los casos. Sin embargo, en esta misma dimensión 54.2 por ciento de los estudiantes evaluaron a sus compañeros en la categoría "distinguida"; caso contrario del profesor, quién evaluó así a 36.6 por ciento de los estudiantes en esta misma categoría.

Por último, en la dimensión de autonomía (trabajo y responsabilidad) los estudiantes evaluaron mucho mejor a sus compañeros (67.1 por ciento), a diferencia del profesor, quien evaluó a 42.2 por ciento de los estudiantes en la categoría "distinguida": una diferencia de 24.9 por ciento. En las Gráficas 4 y 5 se observa que tanto el profesor como los estudiantes evaluaron muy bajo, en la categoría "insatisfactoria", las tres dimensiones del inventario del desempeño académico de los estudiantes: competencia comunicativa, interacción cooperativa y autonomía (trabajo y responsabilidad); particularmente la competencia comunicativa es muy baja en esta categoría ( 0 por ciento el profesor y 1.3 por ciento los estudiantes). Esto nos dice que tanto las evaluaciones del profesor como las de los estudiantes fueron positivas, aunque los estudiantes tendieron a evaluar mejor a sus compañeros de equipo que el profesor.

Gráfica 4. Porcentajes obtenidos en las tres dimensiones del inventario del desempeño académico individual evaluadas por el profesor

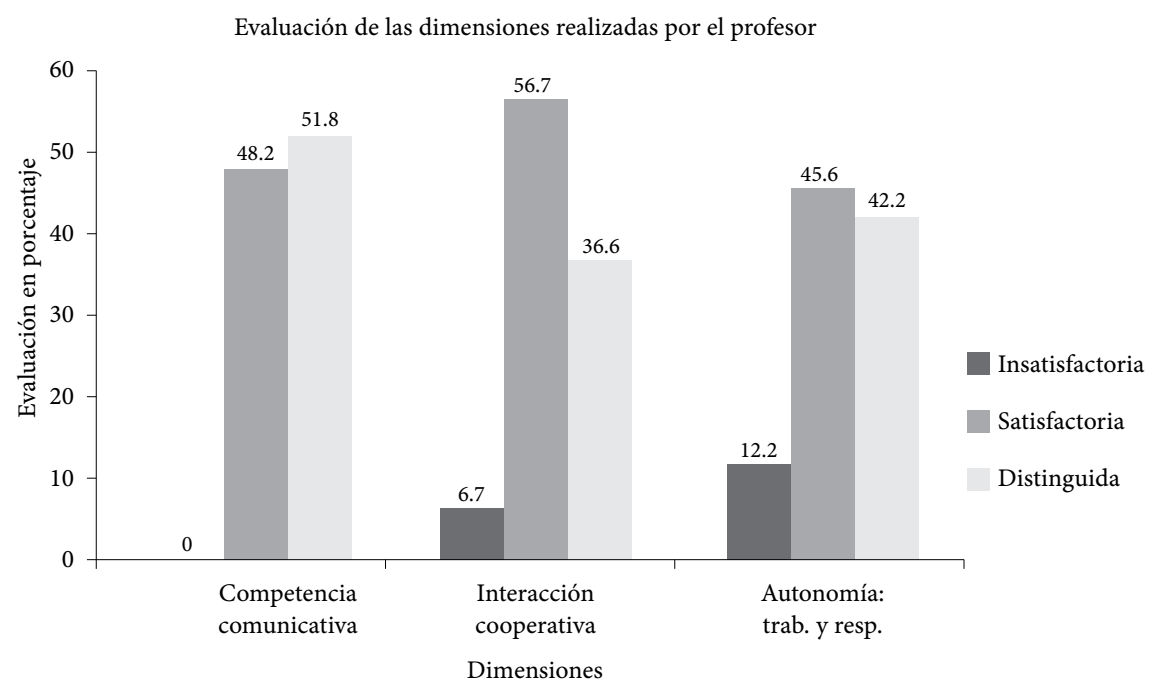


Gráfica 5. Porcentajes obtenidos en las tres dimensiones del inventario del desempeño académico individual evaluadas por los estudiantes

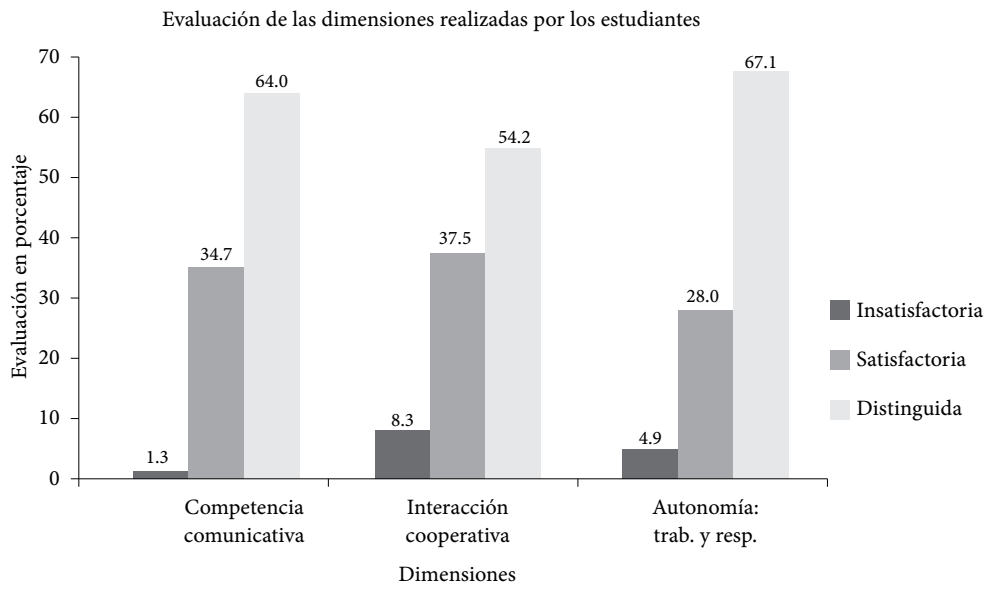

Evaluación de los estudiantes

sobre la estrategia del $A B P$

en el desarrollo de sus habilidades

La mayoría de los estudiantes del grupo experimental evaluaron la estrategia del ABP como positiva y valiosa para el desarrollo del aprendizaje independiente, razonamiento crítico, comunicación interpersonal, procesamiento de la información y formación personal. En la Gráfica 6 se aprecia que éstas obtuvieron en promedio una calificación cercana al 8 (7.98) en todas las dimensiones del diferencial semántico, calificación que se encuentra entre el rango de "fueron de valor" y "fueron de gran valor". Por tanto, se concluye que la evaluación de las actitudes es tan importante como la de los conocimientos, debido a que los estudiantes lograron un mayor autoconocimiento y exploración de sí mismos y de la relaciones con los demás, aspecto crucial para su proceso de desarrollo personal y social (Díaz Barriga y Hernández, 2002).

Gráfica 6. Escala de adjetivos extremos y promedio general para cada ítem del diferencial semántico, aplicado al grupo experimental

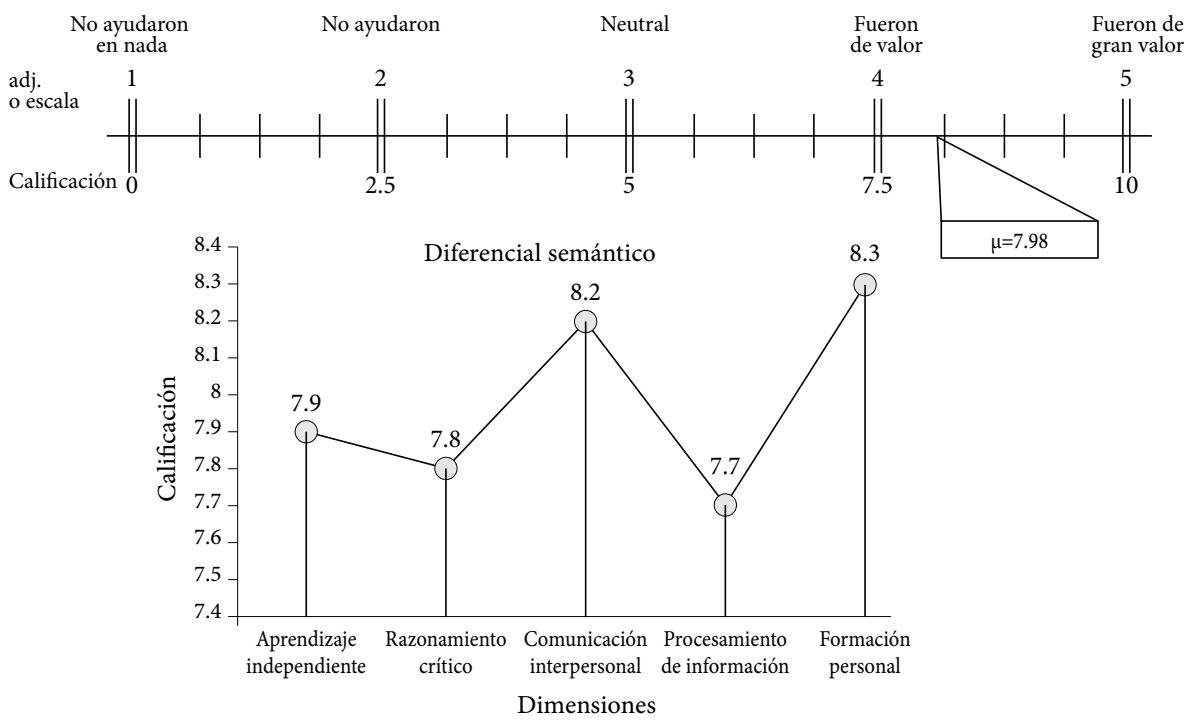


Opiniones de los estudiantes sobre el ABP

La aplicación del cuestionario de opiniones de los estudiantes sobre algunos atributos del contexto durante la aplicación del ABP requirió de un análisis cualitativo de las respuestas; el procedimiento consistió en encontrar patrones generales de respuesta (respuestas similares o comunes) en los discursos de los estudiantes con los que se conformaron las siguientes categorías analíticas, compartidas por la mayoría de los estudiantes:

- Relación entre teoría-práctica. La generalidad de los estudiantes opinaron que las sesiones fueron útiles y relevantes, pues se estableció un nexo entre la teoría y la vida real, que en este caso fue aplicar un problema de salud-enfermedad de relevancia en nuestra sociedad.

- Participación libre de los equipos. También calificaron de interesante el desarrollo de las sesiones, debido a que permitió la participación de los equipos y a que las opiniones vertidas por cada uno se expresaron sin restricciones.

- Promoción de la motivación. En opinión de los estudiantes se generó un clima agradable y por tanto de motivación, en el que mientras se divertían aprendían resolviendo los problemas.

- Colaboración entre los integrantes de los equipos. También expresaron que el ABP promovió la colaboración entre compañeros de equipo, lo cual dio la oportunidad para que aprendieran habilidades de cooperación, tales como tomar decisiones en equipo, ayudarse mutuamente y conocerse, además de que se fomentó la retroalimentación entre pares.

Estos patrones generales de respuestas expresan que la estrategia del $\mathrm{ABP}$ resultó estimulante para los estudiantes, debido a que integró la parte conceptual con un problema real, lo que despertó el interés, y por lo tanto, una motivación para resolver el problema planteado.

\section{Conclusiones}

Los resultados obtenidos permiten observar que el ABP es una herramienta de apoyo pedagógico que se puede utilizar como una estrategia para lograr aprendizajes significativos activos de tipo práctico, en tanto se organiza en torno a la resolución de problemas vinculados al mundo real; además, promueve la motivación al integrar la teoría con la práctica, en nuestro caso, en aspectos biológicos de interés y/o de actualidad para los estudiantes.

Al participar en equipos de forma activa, los estudiantes se motivan y mejoran sus habilidades de integración y transferencia de conocimientos, al mismo tiempo que flexibilizan su pensamiento para resolver por sí mismos los problemas. Cuando se fomenta el trabajo cooperativo en equipo se genera el interés y el compromiso por parte de los estudiantes, pues al ayudarse entre ellos y al resolver entre todos los problemas que se les presentan, se crea apertura e interdependencia. El equipo, por tanto, ayuda a los integrantes a incorporar mecanismos críticos de retroalimentación y comparación con otras formas de pensar que faciliten la posibilidad de cambio permanente.

En tal sentido, el ABP también sirve de apoyo para romper con concepciones espontáneas o del sentido común y fomentar el pensamiento formal de los estudiantes que aún no lo tienen, al tiempo que propicia que quienes ya lo tienen, lo ejerciten. El empleo de esta metodología debe orientarse hacia la creación de formas de pensar que se manifiesten en la manera de hacer las cosas, más que hacia un aprendizaje memorístico, sin que éste se descarte totalmente.

Podemos concluir que el ABP es una alternativa metodológica de enseñanza útil que permite el desarrollo de importantes habilidades cognoscitivas, pero además es una estrategia de 
integración entre los miembros de un equipo, por lo que se recomienda su utilización como un método complementario a la enseñanza de la biología. Esto es: combinar las clases expositivas con el aprendizaje cooperativo y el ABP para cubrir un programa analítico.

La investigación presentada muestra que si bien ambos grupos, el control y el experimental, mejoraron su desempeño académico después de la enseñanza del tema de selección natural, el grupo experimental obtuvo mejores calificaciones aprobatorias y más altas en la post-prueba. Ello nos indica que aun y cuando el titular de la materia en el grupo control empleó técnicas preferentemente constructivistas para la enseñanza del tema, las diferencias alcanzadas en el grupo experimental se deben a las habilidades que desarrollan los estudiantes para solucionar la situación-problema que contempla el ABP como estrategia básica. Ello demuestra que se trata de una alternativa que permite optimizar los procesos de aprendizaje.

Consideramos importante mencionar también la coincidencia de los resultados de esta investigación con lo reportado en otras con propósitos parecidos. Se trata de investigaciones en las que también se encontraron diferencias significativas en el desempeño académico de los estudiantes al aplicar el ABP, tanto en los grupos experimentales como en los grupos control; es decir, tanto en la enseñanza con el método ABP como en la enseñanza con estrategias más tradicionales, el desempeño académico de los estudiantes mejora después de la instrucción; no obstante, la diferencia en cuanto al número de calificaciones aprobatorias en las post-pruebas se encuentra en mayor cantidad en los grupos experimentales (aquellos en los que se aplica el $\mathrm{ABP}$ ), respecto de los grupos control (Fasce et al., 2001; Jiménez et al., 2005; Matus et al., 2005).

Otro rasgo similar encontrado con otras investigaciones es el grado de satisfacción, actitud positiva y disposición que genera entre los estudiantes la aplicación del ABP como estrategia didáctica, en tanto propicia la motivación y por lo tanto crea la necesidad de aprender temas aplicados a su entorno real (Cabalín y Collipal, 2005; Soto et al., 2005); es decir que el ABP resulta tan valioso para el desarrollo de habilidades de pensamiento como para la promoción de actitudes positivas entre los estudiantes. Con ello se confirma que las actitudes son tan importantes como los conocimientos y destrezas académicas adquiridas, en tanto permiten a los estudiantes tener un mayor autoconocimiento y exploración de sí mismos y de las relaciones con los demás.

Es menester mencionar también las ventajas que representan investigaciones como la presentada, en la que se utilizaron métodos y análisis de datos tanto cuantitativos como cualitativos que permitieron describir las tendencias encontradas y compararlas mediante análisis estadísticos para observar la existencia o no de diferencias significativas entre los grupos estudiados; y al mismo tiempo, permiten realizar un análisis más sutil y acabado en cuanto a las ideas, conocimientos y emociones de los sujetos bajo estudio, con lo que se logra complementariedad para el análisis e interpretación de los datos, y con ello mayor validez y confiabilidad de los resultados.

Finalmente, las estrategias didácticas aplicadas en la investigación constituyen una muestra de cómo se pueden llevar a la práctica los principios pedagógicos constructivistas que fundamentan el plan de estudios del $\mathrm{CCH}$, que bien pueden ser aplicados en una variedad de planes y programas en los que se han incorporado las reformas e innovaciones curriculares que colocan al estudiante como constructor activo de su propio aprendizaje.

Del mismo modo, estas estrategias pueden ser elegidas para trabajar temas específicos que a los estudiantes les cuesta trabajo comprender, como por ejemplo "el metabolismo" (enzimas y rutas metabólicas) y "diversidad de los sistemas vivos y metabolismo" (quimioautótrofos, fotoautótrofos y heterótrofos, catabolismo y anabolismo), que se abordan en el quinto semestre del 
$\mathrm{CCH}$. Son temas que requieren un cierto grado de abstracción para su comprensión, por los procesos bioquímicos que se abordan; es por ello que el ABP podría mejorar la comprensión de los estudiantes y la construcción de conocimiento.

\section{REFERENCIAS}

Bisquerra, Rafael (1996), Métodos de investigación educativa, Barcelona, Grupo Editorial Ceac.

Cabalín, Daisy y Collipal, Erika (2005), "Representación a través de redes semánticas naturales del método de enseñanza tradicional y el método de aprendizaje basado en problemas", Revista de Educación en Ciencias de la Salud, vol. 2, núm. 1, en: http://www2.udec.cl/ofem/recs/anteriores/ vol212005/ar21tsiete.htm (consulta: 15 de febrero de 2007).

Coll, César (1997), ¿Qué es el constructivismo?, Buenos Aires, Magisterio del Río de la Plata.

DANIEL, Wayne (1993), Bioestadística: base para el análisis de las ciencias de la salud, México, Uthea Noriega Editores.

De Manuel, Jordi y Ramón Grau (1996), "Concepciones y dificultades comunes en la construcción del pensamiento biológico", Alambique: Didáctica de las Ciencias Experimentales, núm.7, pp. 71-82.

DíAz Barriga, Frida (2006), Enseñanza situada. Vínculo entre la vida y la escuela, México, Mc Graw-Hill.

DíAz Barriga, Frida y Gerardo Hernández (2002), Estrategias docentes para un aprendizaje significativo: una interpretación constructivista, México, Mc Graw-Hill.

Dytнам, Calvin (2001), Choosing and Using Statistics: A biologist's guide, Londres, Blackwell Science, en: wikipedia.org/wiki/ Lluvia_de_ideas (consulta: 28 agosto de 2007).

Estévez, Etty (2002), ¿Qué es la enseñanza y qué es el aprendizaje?, Madrid, Paidós.

ETXEBERRIA, Juan, Luis Joaristi y Luis Lizasoain (1991), Programación y análisis estadísticos básicos con SPSS/PCT, Madrid, Paraninfo.

Fasce, Eduardo, María Calderón, Luis Braga, Manuel De Orúe, Horst Mayer, Heidi Wagemann y Soledad Cid (2001), "Utilización del aprendizaje basado en problemas en la enseñanza de física en estudiantes de medicina”, Revista Médica de Chile, vol. 129, núm. 9, en: www.cielo.cl/scielo.php?pid= S0034... scipt $=$ sci (consulta: 13 de noviembre de 2006).

Hernández, Roberto, Carlos Fernández y Pilar Baptista (2003), Metodología de la investigación, México, Mc Graw-Hill.
Instituto Tecnológico y de Estudios Superiores de Monterrey (ITESM) (2004), Las estrategias y técnicas didácticas en el rediseño, México, ITESM-Dirección de Investigación y Desarrollo Educativo.

Jiménez, L., J.P. Miranda, J. Quilodrán y J. Valdés (2005), "Neuroanatomía basada en aprendizaje en base a problemas (ABP)", trabajo presentado en el III Congreso Internacional de Educación en Ciencias de la Salud, Santiago de Chile, en: Revista de Educación en Ciencias de la Salud, vol. 2, núm. 2 (resúmenes del congreso), en: http://www2.udec.cl/ ofem/recs/anteriores/vol222005/ar22treintcuatro.htm (consulta: 13 de noviembre de 2006).

Lomelí, Guadalupe (1991), “Acerca de la enseñanza de la biología”, Revista de la Educación Superior, vol. XX, núm. 77, pp. 117-130.

Matus, Olga, Sylvia Palacios, Alejandro Soto y Eduardo Fasce (2005), "Utilización del aprendizaje basado en problemas en la enseñanza de informática básica”, Revista Médica de Chile, vol. 21, en: www.udec.cl/ofem/recs/ anteriores/vol212005/artcong05.htm (consultado: 15 de febrero de 2007).

Monereo, Carles (coord.) (1999), Estrategias de enseñanza y aprendizaje, Barcelona, Graó.

Morales, Patricia y Victoria Landa (2004), "Aprendizaje basado en problemas", Theoría, vol. 13, pp. 145-147.

Morrone, Juan (2005), Sistemática, biogeografía, evolución: los patrones de la biodiversidad en tiempo-espacio, México, UNAM-Las prensas de ciencias.

Oficina de Educación de California (EUA) (2002), “The Multimedia Project. Aprendizaje basado en proyectos con multimedios", documento traducido y adaptado por Jaime Sánchez I., en: http://mmpchile.c5.cl/pag/utiles/metod. doc (consulta: 27 de diciembre de 2008).

Olivares, Sergio (2001), "El aprendizaje basado en problemas. Una propuesta metodológica para transformar la universidad", Universidad Autónoma de Nayarit, en: http:// www.congresoretosyexpectativas.udg.mx/ Congreso\%202/Mesa\%201/d)\%20Aprendizaje\%20asistido\%20profesional/1.d.4..pdf (consulta: 4 de agosto de 2007). 
Pozo, Juan Ignacio (1993), "Estrategias de aprendizaje”, en César Coll, Jesús Palacios y Álvaro Marchesi (comp.), Desarrollo psicológico y educación II. Psicología de la educación, Madrid, Alianza, pp. 199-221.

Soтo, Alejandro, Sylvia Palacios, Pilar Ibáñez, Olga Matus y Eduardo Fasce (2005), "Evaluación por los internos de medicina, de la metodología de aprendizaje basado en problemas utilizada en primer y segundo año de la carrera", vol. 21, en: www.udec.cl/ofem/recs/ anteriores/vol212005/artcong05.htm (consulta: 15 de febrero de 2007).
STAKE, Robert (1999), Investigación con estudios de casos, Madrid, Morata.

UNAM-Colegio de Ciencias y Humanidades (CCH) (1996), Plan de Estudios Actualizado (PEA), México, UNAM-CCH-Unidad Académica del Ciclo de Bachillerato, 25 aniversario.

UNAM-Colegio de Ciencias y HumanidadesNaucalpan (2004), Jornada de balance académico, Naucalpan, UNAM-CCH.

UNAM-Colegio de Ciencias y HumanidadesDirección General (2006), Orientación y sentido de las áreas del Plan de Estudios Actualizados, México, UNAM-CCH. 